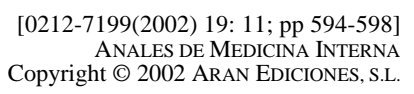

Copyright () 2002 ARAN EDICIONES, S.L.

An. Med InTERnA (Madrid)

Vol. 19, N. ${ }^{\circ} 11$, pp. 594-598, 2002

\title{
Tratamiento de la enfermedad de Behçet
}

\author{
A. VIDALLER PALACÍN, J. ROBERT OLALLA, B. SANUY JIMÉNEZ, \\ G. RUFI RIGAU, J. FOLCH CIVIT*, A. CHARTE GONZÁLEZ \\ Departamento de Medicina Interna. Unidad de Diagnóstico y Seguimiento de \\ Inmunología Clínica y Alergias. *Departamento de Oftalmología. Institut Universitari \\ Dexeus. Barcelona
}

THERAPY REVISION OF BEHÇET'S DISEASE

\section{RESUMEN}

La enfermedad de Behçet, proceso inflamatorio de etiología desconocida, suele presentarse en forma de aftas orales recurrentes, úlceras genitales, uveítis y lesiones cutáneas. De todas formas su espectro clínico es amplio y puede afectar a otros órganos y sistemas como el sistema nerviosos central, articulaciones, vasos sanguíneos y el aparato digestivo. Su aproximación terapéutica es compleja y diferente en función de los distintos órganos afectados.

En la aftosis compleja se suele iniciar el tratamiento con fármacos tópicos, colchicina y dapsona. La utilización de talidomida, prednisona por vía oral y metotrexate se reserva para casos en que no hay respuesta a los fármacos anteriores.

En la afectación ocular severa y en la afectación sistémica el tratamiento es más agresivo y suele combinar corticoides con fármacos inmunosupresores, siendo los más utilizados la ciclosporina, azatioprina, ciclofosfamida, interferón-alfa-2a y el clorambucil.

PALABRAS CLAVE: Enfermedad de Behçet. Ulceras orales. Aftas genitales.

\begin{abstract}
Behçet's disease is an inflammatory process of unknown origin, which usually presents with recurrent oral ulcers, genital aphthae, uvei tis and cutaneous lesions. However, a wide variety of clinical manifesta tions have been reported, and virtually any organ system may be affected, showing central nervous system, joints, blood vessels or gastrointestinal tract involvement. Therapeutic approach remains com plex, and varies in basis of the affected organs.

Complex aphthosis may respond to topical therapy, colchicine and dapsone. If this therapy does not result in adequate disease control, tha lidomide, oral prednisone and methotrexate may be useful.

When severe ocular lesions or systemic manifestations are present. therapies tend to be more aggressive, usually combining corticosteroids with immunosuppressive agents as cyclosporin, azathioprine, cyclop hosphamide, interferon-alfa-2a, and chlorambucil.
\end{abstract}

KEY WORDS: Behçet disease. Oral ulcer. Genital aphthae.

Vidaller Palacín A, Robert Olalla J, Sanuy Jiménez B, Rufi Rigau G, Folch Civit J, Charte González A. Therapy revision of Behçet's disease. An Med Interna (Madrid) 2002; 19: 594-598.

La enfermedad de Behçet (EB), descrita por Hulusi Behçet en 1937, es un proceso inflamatorio de etiología desconocida que se caracteriza por aftas orales recurrentes, úlceras genitales, uveítis y lesiones cutáneas (1). Menos frecuentes son otras manifestaciones a nivel sistémico como la afectación gastrointestinal, del sistema nervioso central, vasculares o las articulares. El International Study Group for Behçet's Disease estableció en 1990 como criterios diagnósticos para la enfermedad de Behçet la existencia de ulceración oral recurrente con al menos dos de las siguientes manifestaciones clínicas: ulceración genital recurrente, afectación ocular, afectación cutánea o test de patergia positivo (2) (Tabla I).

El tratamiento de la enfermedad de Behçet depende del tipo de manifestación clínica y de la gravedad de la misma. El amplio espectro clínico de esta enfermedad aconseja un manejo multidisciplinario. Hasta el momento el escaso número de ensayos terapéuticos controlados, así como el curso variable de la enfermedad, dificulta la estandarización terapéutica.

A continuación proponemos una revisión terapéutica de las diferentes manifestaciones de la enfermedad de Behçet.

\section{AFTAS ORALES Y GENITALES}

La aftosis oral se presenta como una lesión de bordes bien definidos, rodeada de halo eritematoso y con un fondo amarillento. Variables en tamaño y número, suelen ser dolorosas, y afectan habitualmente a cara interna de los labios, mejillas y

Trabajo aceptado: 5 de marzo de 2002

Correspondencia: Ángel Charte González. Departamento de Medicina Interna. Institut Universitari Dexeus. C/ Calatrava, 83. 08017 Barcelona. 


\section{TABLA I}

CRITERIOS DIAGNÓ STICO S PARA LA ENFERM EDAD DE BEHÇET

Úlceras orales recurrentes

Aftas menores, aftas mayores o úlceras herpetiformes observadas por el médico o el paciente, con un mínimo de tres episodios durante un periodo de 12 meses

Más dos de los siguientes:

Úlceras genitales recurrentes

Ulceras o cicatrizaciones aftosas observadas por el médico o el paciente

Lesiones oculares

Uveítis anterior o posterior, presencia de células en el vítreo al examen con lámpara de hendidura: o bien vasculitis retiniana diagnosticada por el oftalmólogo

\section{Lesiones cutáneas}

Eritema nodoso observado por el médico o por el paciente, pseudofoliculitis, o lesiones pápulo-pustulosas; o nódulos acneiformes observados por el médico en pacientes postadolescentes no tratados con corticoesteroides

\section{Análisis de patergia positivo}

Evaluado por un médico a las 48 horas

International Study Group for Behçet's Disease. Lancet 1990; 335: $1078-80$

borde de la lengua. Suelen regresar en unas dos semanas y curan sin dejar cicatriz.

Las aftas genitales, de aspecto similar a las aftas orales, suelen dejar cicatriz. En los hombres se localizan en el escroto $\mathrm{y}$ pene, y en la mujer en labios mayores y menores.

Como tratamiento tópico son efectivos los colutorios ora les con cantidades variables de Clorhexidina, Difenhidramina, o Hidrocortisona que mejora el dolor de las aftas orales recurrentes. La solución de Lidocaína al $2 \%$ es especialmente útil como tratamiento sintomático en pacientes con dificultad para la ingesta por el dolor. Los corticoides tópicos o intralesiona les se han mostrado útiles en el tratamiento de las aftas orales y genitales (3). Un reciente estudio a doble ciego ha demostrado que el Sucralfato en suspensión es eficaz para el tratamiento del dolor de las aftas orales y genitales en estos pacientes (4).

El tratamiento sistémico, se ha utilizado para el control y tratamiento de las aftas orales, en paciente con EB, por vía oral.

La utilización de Colchicina por vía oral a dosis de $0,6 \mathrm{mg} / 8$ horas, aunque con una efectividad variable y respuesta en sólo $50 \%$ de los casos, más en mujeres, se ha demostrado disminuye el tamaño, la frecuencia y la duración de las aftas (3).

La Dapsona a dosis de $100 \mathrm{mg} /$ día es eficaz como tratamiento oral de la EB. Antes de empezar el tratamiento con Dapsona es preciso descartar la existencia de un déficit de glucosa-6-fosfato deshidrogenasa (G-6PDH). Se recomienda reducir la dosis a $50 \mathrm{mg} /$ día si aparece descenso de la hemoglobina (3).

También son útiles los corticoides orales, a dosis de 10$20 \mathrm{mg}$ al día, aunque suele reservarse para casos con refractariedad a la Colchicina.

Han aparecido diversos estudios que inciden en la eficacia, en el control de la aftosis oral recurrente y en la aftosis de la EB, de la Pentoxifilina. Las dosis utilizadas son de $600 \mathrm{mg} /$ día en dos dosis $(5,6)$.

En el manejo de la aftosis severa se puede utilizar: la Talidomida, el Metotrexate y el Interferón- $\alpha-2 a$. El principal inconveniente del tratamiento de la aftosis oral y genital con estos fármacos es el alto índice de recidiva cuando se suspende el tratamiento (7).

El Metotrexate es utilizado a dosis de $7-20 \mathrm{mg}$ a la semana. Los efectos secundarios más frecuentes son las náuseas, astenia, alteración del perfil hepático, y de forma rara la neumonitis, leucopenia y trombocitopenia. Su utilización está contraindicada en pacientes con enfermedad hepática o insuficiencia renal (3).

El Interferón- $\alpha$ - $2 a$ se usa a dosis de 9 millones de unidades subcutáneas tres veces por semana durante tres meses y dosis posteriores de mantenimiento de 3 millones de unidades subcutáneas tres veces por semana, en la aftosis, en la vasculitis pustulosa y en la artritis. En el estudio de Zouboulis y cols., sobre 144 casos, la mejoría de las manifestaciones mucocutáneas es evidente en $74 \%$ de los pacientes, y de las manifestaciones articulares en el $93 \%$ de los pacientes. De todas formas el número de recurrencias dentro de los siete primeros meses después de suspender el tratamiento es del $38 \%$. Los efectos secundarios más habituales son leucopenia reversible, cuadros influenza-like transitorios, psicosis e hipertiroidismo (8).

La Talidomida estaría indicada en varones y mujeres en edad fértil histerectomizadas o con ligadura de trompas bilateral. Las dosis utilizadas son de $100 \mathrm{mg}$ al día con dosis máximas de 300mg al día. Como efectos secundarios, además de la capacidad teratogénica, se han descrito casos de somnolencia, eritema nodoso-like y la polineuropatía $(7,11)$.

\section{MANIFESTACIONES CUTÁNEAS}

Las manifestaciones cutáneas más comunes son el eritema nodoso y la pseudofoliculitis que se manifiesta como lesión inicialmente papulosa, sobre la que aparece pústula estéril y se cubre posteriormente de costra. Las manifestaciones vasculares pueden expresarse en la piel en forma de vasculitis leucocitoclástica, pustulosa o linfocítica.

Aunque algunos autores han recomendado tratamiento tópico con corticoides se suele utilizar el tratamiento sistémi co por vía oral. Existe una amplia experiencia en el manejo con Colchicina en la vasculitis necrotizante; y a partir de estos estudios algunos autores han presentado series de tratamiento en que las manifestaciones cutáneas de la EB son controladas de forma satisfactoria con este fármaco. La mayoría de series publicadas coinciden en que la Colchicina a dosis de 0,5 $\mathrm{mg} / 8 \mathrm{~h}$ es el tratamiento de elección de las manifestaciones cutáneas de la EB (9-10).

En pacientes en que se descarte un déficit de G-6PDH se puede utilizar la Dapsona a dosis de $100 \mathrm{mg} /$ día; en caso de aparecer anemia se pueden reducir la dosis a $50 \mathrm{mg}$ /día (3). 
Los glucocorticoides a dosis de $0,5 \mathrm{mg} / \mathrm{kg} /$ día se han mostrado igualmente eficaces.

Existen distintas series publicadas que han mostrado la eficacia del tratamiento con Talidomida para el control de las manifestaciones cutáneas, especialmente la pseudofoliculitis, a dosis de $100 \mathrm{mg} /$ día (11).

\section{MANIFESTACIONES ARTICULARES}

Las manifestaciones articulares, presentes en más del $50 \%$ de los pacientes, suelen presentarse como monoartritis o poliartritis, de características inflamatorias, no erosivas. Las articulaciones habitualmente afectadas son rodillas, tobillos, codos y muñecas.

El tratamiento de las manifestaciones articulares se basa en el reposo y en la utilización de analgésicos y antiinflamatorios. En pacientes en los cuales la artritis es resistente al tratamiento con antiinflamatorios no esteroideos (AINEs) se ha utilizado la Colchicina, la Sulfasalazina, dosis bajas de Corticoides, el Interferon- $\alpha$-2-a y la Azatioprina con buenos resultados (12).

\section{MANIFESTACIONES OCULARES}

La uveítis posterior y la vasculitis retiniana son las manifestaciones oculares más frecuentes de la EB. La uveítis anterior con hipopion (presencia de pus en la cámara anterior del ojo) es la manifestación clásica en las descripciones iniciales de la EB. La recurrencia y gravedad de las manifestaciones oculares lleva a un número importante de pacientes a la ceguera lo que obliga a un tratamiento enérgico y agresivo de esta patología.

En la uveítis anterior son útiles los tratamientos tópicos con agentes midriáticos y corticoesteroides locales. La Col chicina, que viene siendo utilizada para el tratamiento de las manifestaciones cutáneo-mucosas y articulares de la EB, se ha mostrado eficaz en la prevención de la uveítis anterior y posterior. La Azatioprina también se ha mostrado eficaz para controlar los brotes de uveítis anterior (12).

En las manifestaciones oculares graves se viene utilizando corticoides sistémicos a dosis de $1 \mathrm{mg} / \mathrm{Kg} /$ día. Se ha mostrado su efecto paliativo en la fase aguda pero no mejora el pronóstico ni previene las complicaciones oculares como la ceguera por lo que no se recomienda su utilización como fármaco único.

La utilización de fármacos citotóxicos como Clorambucil, Ciclofosfamida o Azatioprina han demostrado eficacia en el tratamiento con remisiones prolongadas y en la prevención de nuevos episodios. No hay estudios definitivos que demuestren qué inmunosupresor es más eficaz en la EB, aunque el más documentado es la Azatioprina. El principal inconveniente es el desarrollo de infertilidad en tratamientos prolongados, las infecciones oportunistas y las aberraciones cromosómicas que pueden conducir al desarrollo de neoplasias $(13,14)$.

La Ciclosporina $A$ que tiene una acción más rápida que otros inmunosupresores, se ha mostrado especialmente eficaz en pacientes con lesiones oculares refractarias al tratamiento convencional con Colchicina, corticoides, Ciclofosfamida o Azatioprina (15). Inicialmente se utilizaban dosis de 10 $\mathrm{mg} / \mathrm{kg} /$ día con importantes limitaciones por su alta nefrotoxi- cidad; actualmente se recomiendan dosis de $5 \mathrm{mg} / \mathrm{Kg} / \mathrm{día}$ con asociación a corticoides. Otros efectos secundarios descritos son la hiperglicemia y la hipertensión arterial. Está contraindicado en aquellos pacientes con EB que hayan presentado manifestaciones neurológicas.

La Azatioprina en asociación a Ciclosporina A y corticoides muestra tasas de remisión considerables, que puede llegar hasta el $66 \%$ de los casos. Por esta razón está siendo la fórmula terapéutica aconsejada por un número importante de autores.

Existen diversos estudios recientes que demuestran la eficacia del Tacrolimus (FK506) en pacientes con uveítis posterior refractaria al tratamiento con Ciclosporina (16).

El Interferón- $\alpha-2 a$ es la alternativa terapéutica en casos de uveítis posterior corticoresistente. Se ha demostrado que mejora el edema y celularidad del vítreo. Se utiliza a dosis iniciales de 9 millones de unidades tres veces a la semana durante un periodo de tres meses, posteriormente las dosis de mantenimiento son de 3 millones de unidades tres veces a la semana. Con estas pautas de tratamiento se han observado remisiones completas en un $67 \%$ de los pacientes durante cuatro meses (8). Sigue siendo una limitación importante el alto índice de recidiva precoz al retirar el fármaco; además de la ausencia de consenso en cuanto a dosis, y el riesgo de retinopatía isquémica. En cuanto a efectos secundarios se han descrito leucopenia reversible, cuadros influenza-like transitorios, psicosis e hipertiroidismo.

En casos de vasculitis retiniana no controlada se vienen utilizando bolus de Metilprednisolona a dosis de 1gramo endovenoso al día durante tres días (17).

\section{MANIFESTACIONES NEUROLÓGICAS}

Las manifestaciones neurológicas suelen ser por afectación inflamatoria del sistema nervioso central, de forma menos frecuente por afectación vascular a nivel local, o por polineuropatía periférica.

Las formas más frecuentes de presentación son la meningitis o meningoencefalitis, que cursa con líquido de características inflamatorias no infecciosas. Los déficits neurológicos suelen ser en forma de trastornos sensitivos, síndrome piramidal, convulsiones, síndrome cerebeloso, síndrome vestibular y parálisis oculomotoras. La trombosis de pequeños vasos cerebrales o de grandes senos venosos se traduce por hipertensión endocraneal. La polineuritis es poco frecuente. Se han descrito cuadros confusionales, síntomas psiquiátricos y casos de demencia (18-20).

En las manifestaciones neurológicas por afectación vascular se recomienda el tratamiento anticoagulante. El tratamiento específico de las manifestaciones vasculíticas se desarrolla en un apartado específico.

En la afectación del sistema nervioso central se recomienda la asociación de corticoides (a dosis de $1 \mathrm{mg} / \mathrm{kg} / \mathrm{día}$ ) con agentes inmunosupresores. La utilización de corticoides como terapia única se ha demostrado insuficiente y con un papel meramente paliativo (18). Los inmunosupresores más habituales son la Ciclofosfamida a dosis de $2 \mathrm{mg} / \mathrm{kg} / \mathrm{día}$ o en "bolus" de $1 \mathrm{~g}$, la Azatioprina a dosis de $2 \mathrm{mg} / \mathrm{kg} / \mathrm{día}$ y el Clo rambucil a dosis de 0,1-0,2 mg/kg/día (12,23-24).

Otra alternativa es la Metilprednisolona en bolus de $1 \mathrm{~g}$ durante 3 días con Clorambucil a dosis de $0,1 \mathrm{mg} / \mathrm{kg} /$ día con dosis máximas de $8 \mathrm{mg}$ /día. 
TABLA II

M O DALIDADES TERAPÉUTICAS DE LAS M ANIFESTACIO NES CLÍNICAS DE LA ENFERM EDAD DE BEHÇET

\begin{tabular}{|c|c|c|}
\hline & Formas leves y moderadas & Formas severas \\
\hline Aftosis oral y genital & $\begin{array}{c}\text { colutorios orales } \\
\text { Corticoides tópicos o intralesionales } \\
\text { Sucralfato } \\
\text { Colchicina } \\
\text { Corticoides sistémicos } \\
\text { Dapsona }\end{array}$ & $\begin{array}{c}\text { Talidomida } \\
\text { M etotrexate } \\
\text { Interferon- } \alpha-2 a\end{array}$ \\
\hline Lesiones cutáneas & $\begin{array}{c}\text { Colchicina } \\
\text { Dapsona } \\
\text { Talidomida } \\
\text { Corticoides sistémicos }\end{array}$ & M etotrexate \\
\hline Afectación articular & AINES & $\begin{array}{c}\text { Colchicina } \\
\text { Sulfosalazina } \\
\text { Corticoides } \\
\text { Azatioprina } \\
\text { Interferon- } \alpha-2 a\end{array}$ \\
\hline Lesiones oculares & $\begin{array}{c}\text { Corticoides tópicos } \\
\text { Corticoides intraoculares } \\
\text { Corticoides sitémicos } \\
\text { Colchicina }\end{array}$ & $\begin{array}{c}\text { Ciclosporina } \\
\text { Clorambucil } \\
\text { Ciclofosfamida } \\
\text { Azatioprina } \\
\text { Interferon- } \alpha-2 a \\
\text { Tacrolimus (FK506) }\end{array}$ \\
\hline Sistema nervioso central & $\begin{array}{c}\text { Corticoides sistémicos } \\
\text { Clorambucil } \\
\text { Ciclofosfamida } \\
\text { Azatioprina }\end{array}$ & \\
\hline Afectación vascular & $\begin{array}{l}\text { Antiagregantes } \\
\text { Anticoagulantes } \\
\text { Cirugía vascular }\end{array}$ & $\begin{array}{c}\text { Corticoides sistémicos } \\
\text { Ciclofosfamida } \\
\text { Azatioprina }\end{array}$ \\
\hline Sistema gastrointestinal & Sulfasalazina & $\begin{array}{c}\text { Corticoides sistémicos } \\
\text { Cirugía }\end{array}$ \\
\hline
\end{tabular}

\section{MANIFESTACIONES VASCULARES}

Las manifestaciones vasculares pueden presentarse como tromboflebitis superficial, como trombosis venosa o como arteritis. La arteritis se manifiesta por fenómenos oclusivostrombóticos y aneurismáticos que afectan de forma preferente aorta, arteria pulmonar, arteria poplítea, arteria femoral, arteria subclavia y menos frecuente carótida común; y que son responsables de fenómenos infárticos o hemorrágicos sobre diferentes órganos.

El tratamiento de los fenómenos vasculíticos severos se realiza con la asociación de corticoides y agentes inmunosupresores, como en las manifestaciones neurológicas. Se deben contemplar aspectos específicos como el tratamiento antiagregante en la tromboflebitis superficial, el tratamiento descoagulante a largo plazo en la trombosis venosa profunda, el tratamiento quirúrgico con ligadura o injertos o la embolización sobre las oclusiones o aneurismas arteriales (21).
La utilización a largo plazo de corticoides y anticoagulantes se ha demostrado útil para el tratamiento y prevención de las trombosis venosas cerebrales en la EB (22).

\section{MANIFESTACIONES GASTROINTESTINALES}

Las manifestaciones gastrointestinales suelen presentarse en forma de dolor abdominal, diarreas, melenas y ocasionalmente como perforación. La región ileo-cecal es la más frecuentemente afectada, seguida por colon transverso, ascendente y esófago.

Se ha mostrado eficaz el tratamiento con Sulfasalazina a dosis de $2 \mathrm{~g}$ al día. Así mismo, se utilizan para control de formas graves los corticoides sistémicos (12).

La cirugía se reserva para casos de perforación o sangrado persistente. 


\section{CONCLUSIONES}

En pacientes que presenten aftosis compleja debe iniciarse el manejo terapéutico con fármacos tópicos, Colchicina y Dapsona. Si no hay respuesta a estos fármacos se puede utilizar la Talidomida, la Prednisona por vía oral y el Metotrexate.

\section{Bibliografía}

1. Behçet H. Über rezidivierende, aphtose, durch ein virus verursachte Geschwüre am Mund, am Auge und an den Genitalien. Derm Wscmr 1937; 105: 1152-7.

2. International Study Group for Behçet's Disease. Criteria for diagnosis of Behçet's disease. Lancet 1990; 335: 1078-80.

3. Ghate J, Jorizzo JL. Behçet's disease and complex aphthosis. J Am Acad Dermatol 1999; 40: 1-18.

4. Alpsoy E, Hanife ER, Durusoy C, Yilmaz E. The use of Sucralfate suspension in the treatment of oral and genital ulceration of Behçet disease: a randomized, placebo-controlled, double blind study. Arch Dermatol 1999; 135: 529-32.

5. Yasui K, Ohta K, Kobayashi M, Aizawa T, Komiyama A. Successful treatment of Behçet disease with pentoxifylline. Ann Inten Med 1996; 124: 891-3.

6. Pizarro A, Herranz P, García-Tobaruela A, Casado M. Pentoxifilina en el tratamiento de la aftosis orogenital y del síndrome de Behçet. Med Clin (Barc) 2000; 115 (17): 678.

7. Ehrlich G. Behçet disease and the emergence of Thalidomide. Ann Intern Med 1998; 128: 494-5.

8. Zouboulis C, Orfanos C. Treatment of Adamantiades-Behçet disease with systemic Interferon Alfa. Arch dermatol 1998; 134: 1010-6.

9. Miyachi Y, Taniguchi S, Ozaki M, Horio T. Colchicine in the treatmen of the cutaneous manifestation of Behçet disease. British Journal of Dermatology 1981; 104: 67-8.

10. Sais G, Vidaller A, Jucglà A, Gallardo F, Peyrí J. Colchicine in the treatment of cutaneus leukocytoclastic vasculitis. Results of a prospective, Randomized, controled trial. Arch Dermatology 1995; 131: 1399-402.

11. Hamuryudan V, Mat C, Saip S, Özyazgan Y, Siva A, Yurdakul S, et al. Thalidomide in the treatment of mucocutaneous lesions of the Behçet syndrome. Ann Intern Med 1998; 128: 443-50.

12. Sakane T, Takeno M, Suzuki N, Inaba G. Behçet's disease. N Engl J Med 1999; 341 (17): 1284-91.
En la afectación ocular severa y en la afectación sistémica el tratamiento debe combinar corticoides con inmunosupresores. Los inmunosupresores más utilizados son la Ciclosporina, Azatioprina, Ciclofosfamida, Interferon- $\alpha-2$ a y el Clorambucil.

Insistir finalmente en la importancia del manejo multidisciplinario de esta entidad, dado que se trata de una enfermedad sistémica.

13. O'Duffy JD, Robertson DM, Goldstein NP. Chlorambucil in the treatment of uveitis and meningoencephalitis of Behçet's disease. Am J Med 1984; 76: 75-84.

14. Yazici H, Pazarli H, Barnes CG, et al. A controlled trial of azathiorpine in Behçet's disease. N Engl J Med 1990; 322: 282-5.

15. Diaz-Llopis M, Cervera M, Menezo JL. Cyclosporin A treatment of Behçet's disease: a long-term study. Curr Eye Res 1990; 9: 17-23.

16. Sloper CM, Powell RJ, Dua HS. Tacrolimus (FK506) in the treatment of posterior uveitis refractory to cyclosporine. Ophthalmology 1999; 106: 723-8.

17. Reed BJ, Morse LS, Schwab IR. High-dose intravenous pulse Methylprednisolone Hemisuccinate in acute Behçet retinitis. Am J Ophthalmol 1998; 125: 410-1.

18. Farah S, Al-Shubaili A, Montaser A, Hussein JM, Malaviya AN, Mukhtar M, et al. Behçet's syndrome: a report of 41 patients with emphasis on neurological manifestations. J Neurol Neurosurg Psychiatry 1998; 64: 382-4.

19. Banna H, el-Ramahl K. Neurologic involvement in Behçet disease: imaging findings in 16 patients. AJNR Am J Neuroradiol 1991; 12: 791-6.

20. Martínez-Yelamos A, Vidaller A, Mitjavila R, Pujol R, Jato M, Rubio F. Afectación parenquimatosa del sistema nervioso central en la enfermedad de Behçet. Rev Neurol 1998; 27: 223-5

21. Tüzun H, Besirli K, Sayin A, Vural FS, Hamuryudan V, Hizli N, et al Manegement of aneurysms in Behçet syndrome: an analysis of 24 patients. Surgery $1997 ; 121: 150-6$.

22. Wechsler B, Vidailhet M, Piette JC, Bousser MG, Dell Isola B, Blétry $\mathrm{O}$, et al. Cerebral venous thrombosis in Behçet's disease: clinical study and long-term follow-up of 25 cases. Neurology 1992; 42: 614-8.

23. Le Thi Huong Du, Fain O, Wechsler B, Cochereau I, Le Hoang, Souillem J, et al. Intérêt des "bolus" de cyclophosphamide dans la maladie de Behçet. Presse Med 1990; 19: 1355-8.

24. Mateos-Colino A, Gonzalez-Gay MA, Cereijo MJ, Santiago J, Rabuñal R, Brañas F. Neuro-Behcet: estudio evolutivo de cuatro casos tratados con clorambucil. An Med Interna (Madrid) 1995; 12: 600-2. 